\title{
IgG4-related inflammatory pseudotumor of the kidney mimicking renal cell carcinoma: A case report
}

\author{
YI CAI, HAN-ZHONG LI and YU-SHI ZHANG \\ Department of Urology, Peking Union Medical College Hospital, Chinese Academy of Medical \\ Sciences and Peking Union Medical College, Beijing 100730, P.R. China \\ Received February 16, 2015; Accepted March 3, 2016
}

DOI: $10.3892 / \mathrm{ol} .2016 .4408$

\begin{abstract}
IgG4-related disease is a recently recognized clinical entity. It is characterized by diffuse organ swelling or mass formation, a dense lymphoplasmacytic infiltrate rich in IgG4-positive plasma cells with fibrosis and typically an increased serum IgG4 concentration, which may affect various organs. An 80-year-old woman with an otherwise unremarkable previous medical history was revealed to have a renal mass that was indicative of renal malignant carcinoma, for which a radical nephrectomy was performed. The mass was diagnosed as an IgG4-related inflammatory pseudotumor, which was histopathologically confirmed. The patient is currently well without evidence of IgG4-related disease at 3 months post-surgery, and did not require any additional therapy.
\end{abstract}

\section{Introduction}

IgG4-related disease (IgG4-RD) is a newly recognized clinical condition, which was initially described in 2003 as the cause of type 1 autoimmune pancreatitis (1). IgG4-related inflammatory pseudotumors are part of the spectrum of systemic IgG4-RD, which is characterized by diffuse organ swelling or mass formation, a dense lymphoplasmacytic infiltrate rich in IgG4-positive plasma cells with fibrosis and typically an increased serum IgG4 concentration (2). The most common organs involved in IgG4-RD include the pancreas, biliary duct, salivary gland, ocular and lymph nodes, although it has been reported that IgG4-related inflammatory pseudotumors occur in a range of organs $(3,4)$. Renal involvement is uncommon (5). To the best of our knowledge, $<5$ cases of IgG4-related renal inflammatory pseudotumors have been reported in the

Correspondence to: Professor Yu-Shi Zhang, Department of Urology, Peking Union Medical College Hospital, Chinese Academy of Medical Sciences and Peking Union Medical College, 1 Shuaifuyuan Street, Beijing 100730, P.R. China

E-mail: zhangyushi2014@126.com

Key words: immunohistochemistry, inflammatory pseudotumor, kidney, positron emission tomography
English literature (5). The present report describes a case of IgG4-related inflammatory pseudotumor of the kidney mimicking renal malignant carcinoma. Written informed consent was obtained from the patient.

\section{Case report}

An 80-year-old woman was referred to Peking Union Medical College Hospital (Beijing, China) with a low echo-level lesion in the left kidney, which was identified incidentally by abdominal ultrasonography during a routine medical examination. The patient's previous medical history included well-controlled hypertension and asthma, and her medications included amlodipine besylate and an inhaler for asthma. The physical examination and laboratory investigations were normal. Serum creatinine concentration was $63 \mu \mathrm{mol} / 1$ (normal range, $40-84 \mu \mathrm{mol} / \mathrm{l}$ ) and glomerular filtration rate was $91.1 \mathrm{ml} / \mathrm{min} / 1.73 \mathrm{~m}^{2}$ (normal range, $90-120 \mathrm{ml} / \mathrm{min} / 1.73 \mathrm{~m}^{2}$; $35.9 \mathrm{ml} / \mathrm{min} / 1.73 \mathrm{~m}^{2}$ in left kidney; $55.2 \mathrm{ml} / \mathrm{min} / 1.73 \mathrm{~m}^{2}$ in right kidney). Computed tomography (CT; SOMATOM Sensation; Siemens Healthcare, Erlangen, Germany) revealed a mass of $3.9 \times 3.7 \mathrm{~cm}$ located at the inferior portion of the left kidney, with contrast enhancement, which suggested a malignant tumor (Fig. 1A). Subsequent positron emission tomography (PET)-CT (Biograph mCT; Siemens Healthcare) revealed increased ${ }^{18} \mathrm{~F}$-fluorodeoxyglucose uptake of the renal mass (Fig. 1B). Based on the clinical and radiological findings, renal cell carcinoma was diagnosed. A retroperitoneoscopic left radical nephrectomy was performed under the diagnosis of renal cell carcinoma.

Gross examination revealed a $5.0 \times 3.5 \mathrm{~cm}$-sized white homogenous mass that was confined to the kidney (Fig. 2A). Resected tissues were fixed in $10 \%$ neutral-buffered formalin (Beijing Dingguo Changsheng Biotechnology Co., Ltd., Beijing, China), paraffin (Beijing Dingguo Changsheng Biotechnology Co., Ltd.)-embedded and cut into 5-mm sections. The sections were then deparaffinized in xylene, rehydrated in an ethanol series and treated with trypsin (Beijing Dingguo Changsheng Biotechnology Co., Ltd.) and proteases (Beijing Dingguo Changsheng Biotechnology Co., Ltd.). Hematoyxlin and eosin (Beijing Dingguo Changsheng Biotechnology Co., Ltd.) staining was used to observe morphological changes under a microscope (TS100; Nikon Corporation, Tokyo, Japan). Tissue sections were 
A

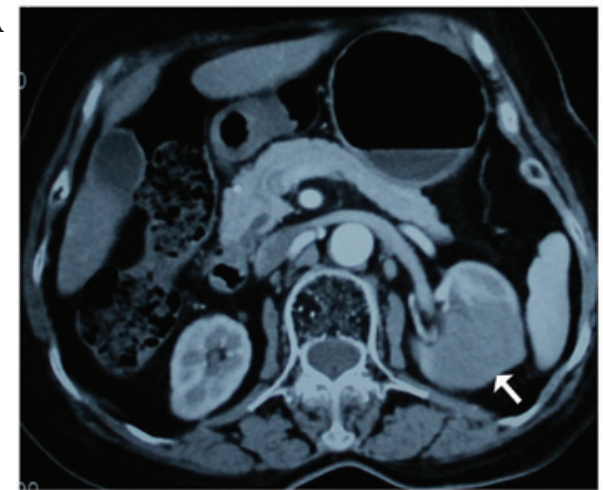

B

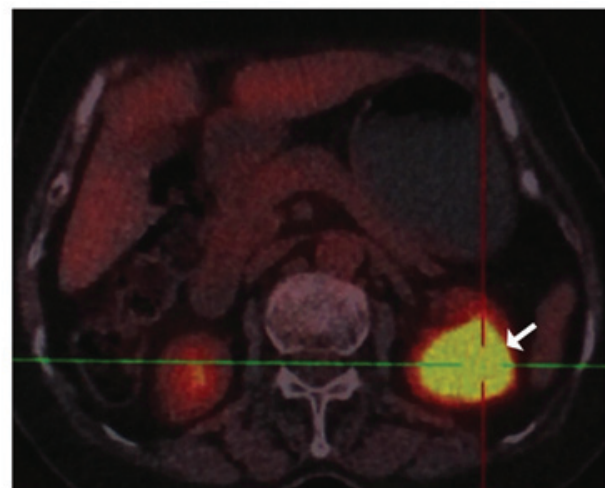

Figure 1. (A) Abdominal CT showing low-density lesions (white arrow) in the left kidney. (B) Positron emission tomography-CT showing a hypermetabolic mass (white arrow) in the left kidney. CT, computed tomography.

A

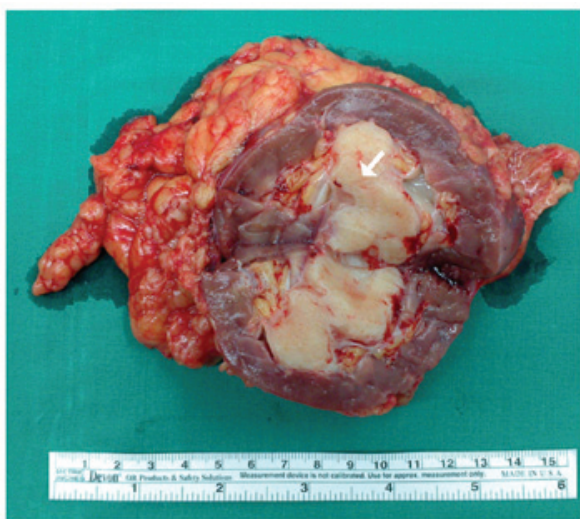

C

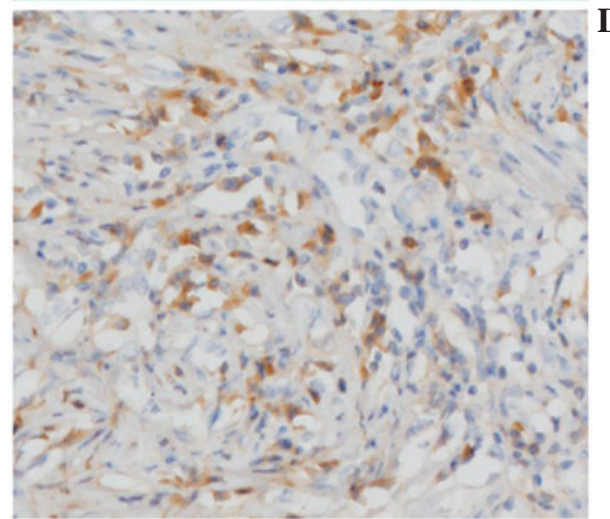

B

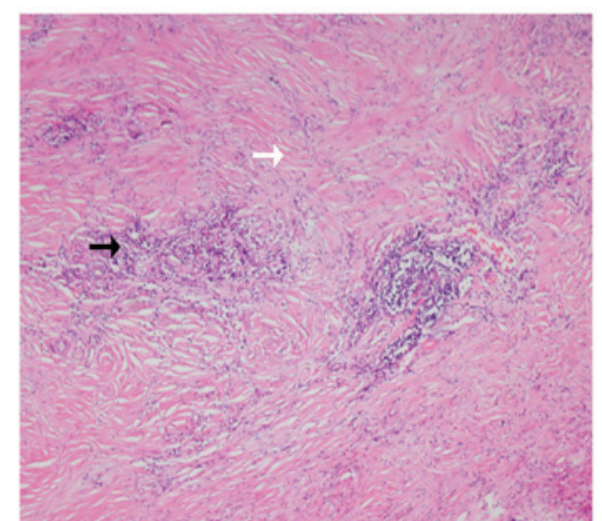

D

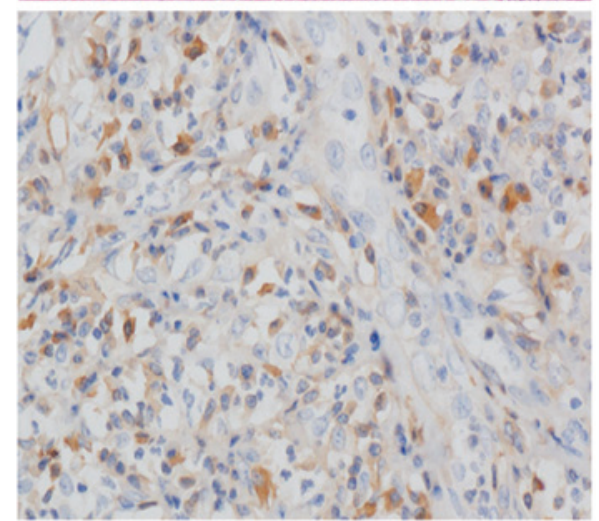

Figure 2. (A) Gross examination of the left kidney showing a 5.0x3.5-cm, white, homogenous mass (white arrow). (B) Histology showing the mass characterized by diffuse lymphoplasmacytic infiltration (black arrow) with myofibroblastic proliferation (white arrow) (staining hematoxylin and eosin; magnification, x100). (C) Anti-IgG antibody staining showing IgG-positive plasma cells (magnification, x200). (D) Anti-IgG4 antibody staining showing IgG4-positive plasma cells (magnification, $\mathrm{x} 200$ ). The IgG4/IgG-positive cell ratio was $>40 \%$. Ig, immunoglobulin.

incubated with monoclonal mouse anti-human CD21 (1:100; cat. no. sc-13135; Santa Cruz Biotechnology, Inc., Dallas, TX, USA), monoclonal mouse anti-human CD3 (1:100; cat. no. sc-20047; Santa Cruz Biotechnology, Inc.), polyclonal goat anti-human CD34 (1:100; cat. no. sc-7045; Santa Cruz Biotechnology, Inc.), monoclonal mouse anti-human CD79 (1:100; cat. no. sc-390372; Santa Cruz Biotechnology, Inc.), monoclonal mouse anti-human S-100 (1:50; cat. no. sc-53438; Santa Cruz Biotechnology, Inc.), polyclonal goat anti-mouse Ki-67 (1:50; cat. no. sc-7846; Santa Cruz Biotechnology, Inc.), polyclonal goat anti-human IgG (1:50; cat. no. sc-34665; Santa Cruz Biotechnology, Inc.) and monoclonal mouse anti-human
IgG4 (1:50; cat. no. sc-69919; Santa Cruz Biotechnology, Inc.) primary antibodies at room temperature for $1 \mathrm{~h}$. The sections were then washed in phosphate-buffered saline (Beijing Dingguo Changsheng Biotechnology Co., Ltd.) and incubated with fluorescein isothiocyanate-conjugated donkey anti-goat IgG (1:240; cat. no. sc-2024; Santa Cruz Biotechnology, Inc.) or horseradish peroxidase-conjugated goat anti-mouse IgG (1:200; cat. no. sc-2005; Santa Cruz Biotechnology, Inc.) secondary antibodies at room temperature for $30 \mathrm{~min}$. Next, sections were stained with ABC solution ((Beijing Dingguo Changsheng Biotechnology Co., Ltd.) and 3,3'-diaminobenzidine (Dako, Carpinteria, CA, USA) and counterstained with 
Mayer's hematoxylin (Beijing Dingguo Changsheng Biotechnology Co., Ltd.). Staining was visualized using a microscope (TS100; Nikon Corporation). Microscopic examination revealed diffuse lymphoplasmacytic infiltration with myofibroblastic proliferation (Fig. 2B). Immunohistochemical staining was positive for anaplastic lymphoma kinase, B-cell lymphoma 2, cluster of differentiation (CD)21, CD3, CD34 (vascular), CD79, S-100, Ki-67, immunoglobulin (Ig)G and the $\mathrm{IgG} / \mathrm{IgG}$-positive cell ratio was $>40 \%$ (Fig. 2C and D). Immunohistochemical staining was negative for desmin, epithelial membrane antigen and smooth muscle actin. The histological and immunohistochemical findings were interpreted to indicate an IgG4-related inflammatory pseudotumor. Serum IgG4 levels were normal $(69.2 \mathrm{mg} / \mathrm{dl}$; normal range, $4.8-105 \mathrm{mg} / \mathrm{dl})$ at two weeks subsequent to surgery. The patient outcome was positive, and there was no evidence of IgG4-RD at 3 months post-surgery. The patient did not undergo any additional therapy.

\section{Discussion}

IgG4-RD is a rare systemic disorder that typically affects middle-aged men, which may involve the pancreas, bile duct, salivary gland, retroperitoneum, ureter, kidney, adrenal gland and prostate, as well as other organs (2). More than $90 \%$ of patients with IgG4-related renal disease have a history of prior extrarenal lesions, including pancreatitis, retroperitoneal fibrosis and sialadenitis (5). In the kidney, the most common form of lesion is tubulointerstitial nephritis; however, various glomerular lesions, in particular membranous glomerulonephritis, may develop simultaneously with tubulointerstitial nephritis (5). IgG4-RD is very rarely observed in the form of an inflammatory pseudotumor of the kidney.

Pathophysiologically, a dense lymphoplasmacytic infiltrate with an increased number of IgG4-positive plasma cells and fibrosis is a key characteristic in IgG4-RD (2). However, the role of IgG4-positive cells remains to be elucidated in IgG4-RD. Approximately $80 \%$ of IgG4-related kidney disease patients demonstrate an increased serum $\mathrm{IgG} 4$ concentration ( $\geq 135 \mathrm{mg} / \mathrm{dl}$; normal range, $4.8-105.0 \mathrm{mg} / \mathrm{dl}$ ). In addition, patients with IgG4-related renal disease typically exhibit hypergammaglobulinemia, hypocomplementemia or mild renal dysfunction $(5,6)$.

It is difficult to discriminate between IgG4-related renal inflammatory pseudotumor and malignant lesions based on imaging studies (5). Abdominal ultrasonography may reveal low echoic lesions or parenchymal swelling, and contrast-enhanced CT frequently reveals isolated or multiple low-density lesions (7). On magnetic resonance imaging (MRI), renal masses demonstrate iso- or hypointensity on T1-weighted images and hypointensity on T2-weighted images, and demonstrate mild contrast enhancement following contrast medium administration (8). The present case was initially approached as renal cell carcinoma due to ultrasound, contrast-enhanced CT and PET-CT findings. To the best of our knowledge, the present case report is the first to include ultrasound, contrast-enhanced $\mathrm{CT}$ and positron emission tomography findings to reveal apparent renal cell tumor-like lesions.

Corticosteroids are the first line treatment regimen for IgG4-RD, although no controlled trial has been performed and the treatment protocol has varied among countries or institutions (9). In the few previous reports of renal involvement with IgG4-RD, the renal lesions have been observed to decrease in size following corticosteroid therapy, with some disappearing and others recurring subsequent to cessation of treatment (10-12). Furthermore, corticosteroids have been reported to improve renal function and decease serum $\operatorname{IgG} 4$ levels after 1 month of therapy; however, renal function may not be totally recovered (10).

The present report described a case of IgG4-related inflammatory pseudotumor of the kidney that was distinguished from a renal malignant tumor. If a subperiosteal tumor-like mass is observed, it is important to consider the possibility of an IgG4-related inflammatory pseudotumor. If the present lesion had been diagnosed as an IgG4-related renal inflammatory pseudotumor prior to surgery, it may have been appropriate to perform a needle biopsy and administer steroid therapy. The present case highlights the importance of recognizing that IgG4-related diseases may involve the kidney and may subsequently result in the development of masses that simulate a renal cell carcinoma.

\section{References}

1. Kamisawa T, Funata N, Hayashi Y, Eishi Y, Koike M, Tsuruta K, Okamoto A, Egawa $\mathrm{N}$ and Nakajima H: A new clinicopathological entity of IgG4-related autoimmune disease. J Gastroenterol 38: 982-984, 2003.

2. Stone JH, Zen Y and Deshpande V: IgG4-related disease. N Engl J Med 366: 539-551, 2012.

3. Yamamoto H, Yamaguchi H, Aishima S, Oda Y, Kohashi K, Oshiro Y and Tsuneyoshi M: Inflammatory myofibroblastic tumor versus IgG4-related sclerosing disease and inflammatory pseudotumor: A comparative clinicopathologic study. Am J Surg Pathol 33: 1330-1340, 2009.

4. Moriarty MA, Dahmoush L and Nepple KG: IgG4 related disease of the ureter (inflammatory pseudotumor). J Urol 191: 1126-1127, 2014.

5. Saeki T and Kawano M: IgG4-related kidney disease. Kidney Int 85: 251-257, 2014.

6. Tsubata Y, Akiyama F, Oya T, Ajiro J, Saeki T, Nishi S and Narita I: IgG4-related chronic tubulointerstitial nephritis without autoimmune pancreatitis and the time course of renal function. Intern Med 49: 1593-1598, 2010.

7. Kuroda N, Nao T, Fukuhara H, Karashima T, Inoue K, Taniguchi Y, Takeuchi M, Zen Y, Sato Y, Notohara K and Yoshino T: IgG4-related renal disease: Clinical and pathological characteristics. Int J Clin Exp Pathol 7: 6379-6385, 2014.

8. Tan TJ, Ng YL, Tan D, Fong WS and Low AS: Extrapancreatic findings of IgG4-related disease. Clin Radiol 69: 209-218, 2014.

9. Kamisawa T, Okazaki K, Kawa S, Shimosegawa T and Tanaka M; Research Committee for Intractable Pancreatic Disease and Japan Pancreas Society: Japanese consensus guidelines for management of autoimmune pancreatitis: III. Treatment and prognosis of AIP. J Gastroenterol 45: 471-477, 2010.

10. Saeki T, Kawano M, Mizushima I, amamoto M, Wada Y, Nakashima H, Homma N, Tsubata Y, Takahashi H, Ito T, et al: The clinical course of patients with IgG4-related kidney disease. Kidney Int 84: 826-833, 2013.

11. Mizushima I, Yamada K, Fujii H, Inoue D, Umehara H, Yamagishi M, Yamaguchi Y, Nagata M, Matsumura M and Kawano M: Clinical and histological changes associated with corticosteroid therapy in IgG4-related tubulointerstitial nephritis. Mod Rheumatol 22: 859-870, 2012.

12. Dhobale S, Bedetti C, Killian P, Ilyas M, Liput J, Jasnosz K and Giclas P: IgG4 related sclerosing disease with multiple organ involvements and response to corticosteroid treatment. J Clin Rheumatol 15: 354-357, 2009. 